\title{
UN CARTÓN Y UN DIBUJO DE CAVALIER D'ARPINO EN MADRID
}

\author{
GonZalo Redín Michaus ${ }^{1}$ \\ Universidad de Alcalá
}

\begin{abstract}
El texto presenta un cartón adquirido en Italia por el XIV duque de Alba (Colección Casa de Alba) atribuido hasta ahora a Fra Bartolomeo, pero que es obra de Giuseppe Cesari, conocido como Cavalier d'Arpino, y está directamente relacionado con el fresco que este pintó en la Colegiata de San Lorenzo de Zagarolo.

Atribuye al pintor un dibujo inédito en la Biblioteca Nacional, a situar durante su etapa juvenil, y realiza algunas consideraciones sobre un folio que tradicionalmente se le han asignado en la misma institución y es más bien obra de su taller. Por último, se identifica una copia, acompañada con el nombre del holandés Cornelis Saftleven, del fresco de la Asunción de María de Cesari en la bóveda de la Capilla Olgiati en Santa Prassede de Roma.

Palabras clave: Cavalier d'Arpino; Giuseppe Cesari; Saftleven; Dibujo; Cartón; Biblioteca Nacional de España; Colección Casa de Alba.
\end{abstract}

\section{A CARTOON AND A DRAWING BY CAVALIER D'ARPINO IN MADRID}

This text presents a cartoon acquired in Italy by the $14^{\text {th }}$ Duke of Alba (Colección Casa de Alba) previously associated with Fra Bartolomeo, but in fact the work of Giuseppe Cesari, known as Cavalier d'Arpino. It is directly related to the fresco he painted in the Colegiata de San Lorenzo de Zagarolo.

The author attributes an anonymous drawing in the Madrid Biblioteca Nacional to Cesari, placing it within an early stage of his career, and offers some considerations on a sheet in the same collection, which has historically been associated with the painter although it is probably a product of his workshop. Lastly, he identifies a copy by the Duch artist Cornelis Saftleven of Cesari's fresco in the dome of the Olgiati Chapel in Santa Prassede in Rome.

Key words: Cavalier d'Arpino; Giuseppe Cesari; Drawing; Saftleven; Cartoon; Biblioteca Nacional de España; Colección Casa de Alba.

Cómo citar este artículo / Citation: Redín Michaus, Gonzalo (2019): "Un cartón y un dibujo de Cavalier d'Arpino en Madrid". En: Archivo Español de Arte, vol. 92, núm. 365, Madrid, pp. 17-24. https://doi.org/10.3989/ aearte.2019.02.

En la sala de pintura italiana del Palacio de Liria en Madrid se muestra un cartón propiedad de la colección Alba que fue adquirido como obra de Fra Bartolomeo en Italia entre 1814 y 1817 por Carlos Miguel Fitz-James Stuart (Madrid 1794-Sion 1835), XIV duque de Alba y VII duque de Berwick (fig. 1) ${ }^{2}$. Esta atribución se ha mantenido hasta hoy, pero se trata de una de los escasas obras en este formato que se conservan de Cavalier d'Arpino (Giuseppe Cesari,

${ }^{1}$ gonzaloredin@hotmail.com / ORCID iD: http://orcid.org/0000-0003-1217-422X.

2 Esta investigación forma parte del proyecto HAR2017-86804-P, Dibujo italiano y español del siglo XVI en la Biblioteca Nacional de España. Agradezco a la Fundación Casa de Alba su inestimable ayuda para poder realizar este trabajo. Colección Casa de Alba. Inv. P. 136. Carboncillo con albayalde diluido, 194 x 139 centímetros. Pita 1959: 52. 


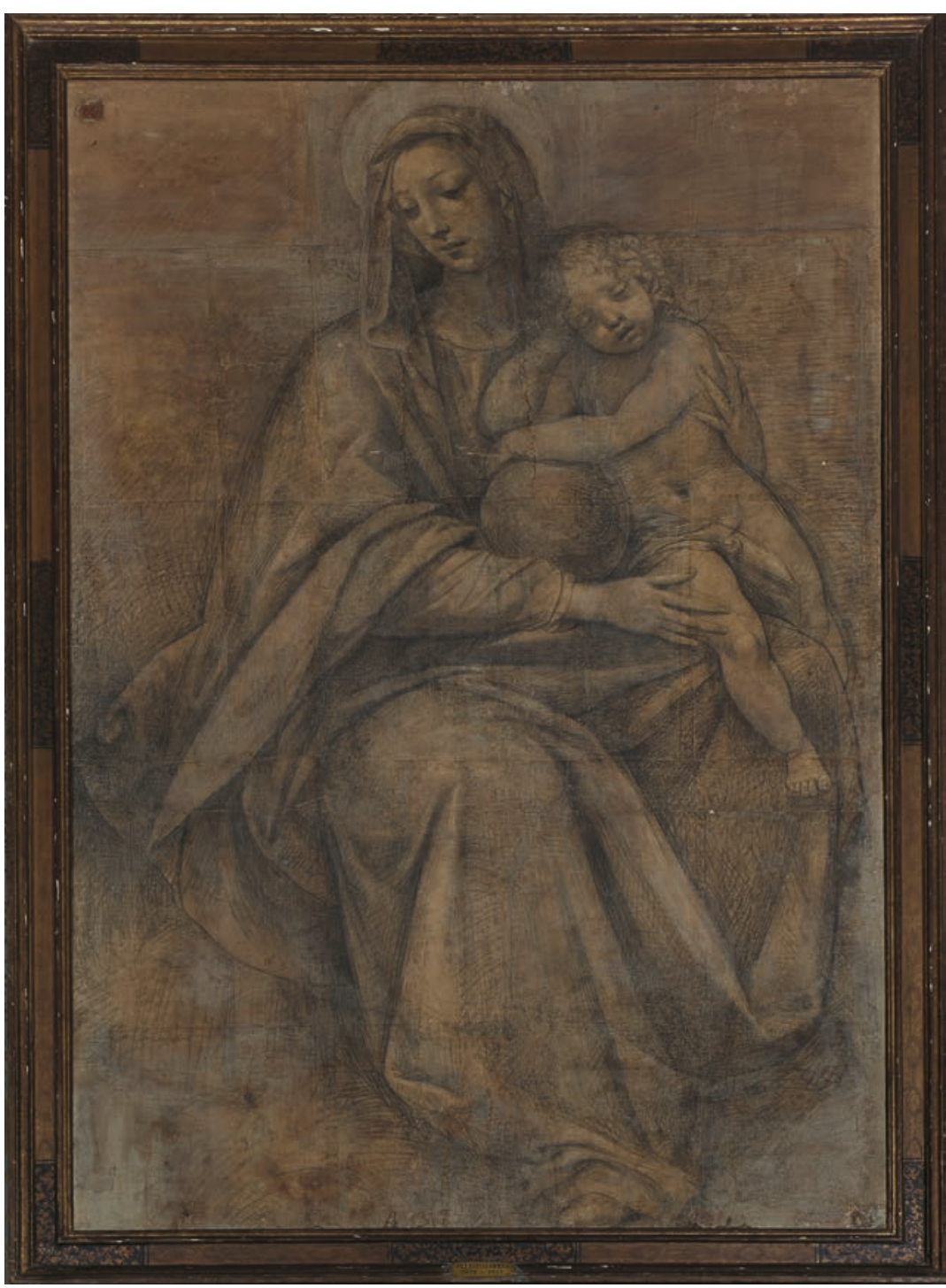

Fig. 1. Giuseppe Cesari

Virgen con el Niño dormido. Colección Casa de Alba. Palacio de Liria. Madrid.

1568-1640) ${ }^{3}$, y muy interesante, dadas sus dimensiones y el hecho de que ofrece una composición completa que fue empleada en buena medida en el fresco, recientemente restaurado, de la Virgen con el Niño dormido de la segunda capilla izquierda de la Colegiata de San Lorenzo en Zagarolo, Roma (fig. 2).

Es probable que el dibujo se comprara en Florencia, pues figura como "Un gran cartón. Virgen con el niño del Frate" en una lista fechada 1817 que recoge parte de la colección del duque que debía ser enviada desde la capital toscana a Livorno, y desde allí a España ${ }^{4}$, donde el coleccionismo de obra gráfica era raro, salvo en el círculo de los monarcas. Esta compra reflejaba la decidida vocación internacional de la colección del noble, en la que tuvieron cabida los primitivos italianos, de los habría tenido pocas noticias antes de llegar Paris en marzo

3 Sobre sus cartones, Röttgen 1973: 161; 2002: 325-326 y 353-364; 2012-2013, II: 420 y III: 24-26. Bolzoni, 2013: 310.

${ }^{4}$ Este elenco, sin fecha, forma parte de un documento más amplio de 1817, que recoge otras obras suyas que se encontraban en Roma y Nápoles, y que también debían ser enviadas a Livorno para luego ser remitidas a España. Effetti incassati a Firenze e spediti a Livorno, 1817, Archivo Casa de Alba (ADA), 157.46. 


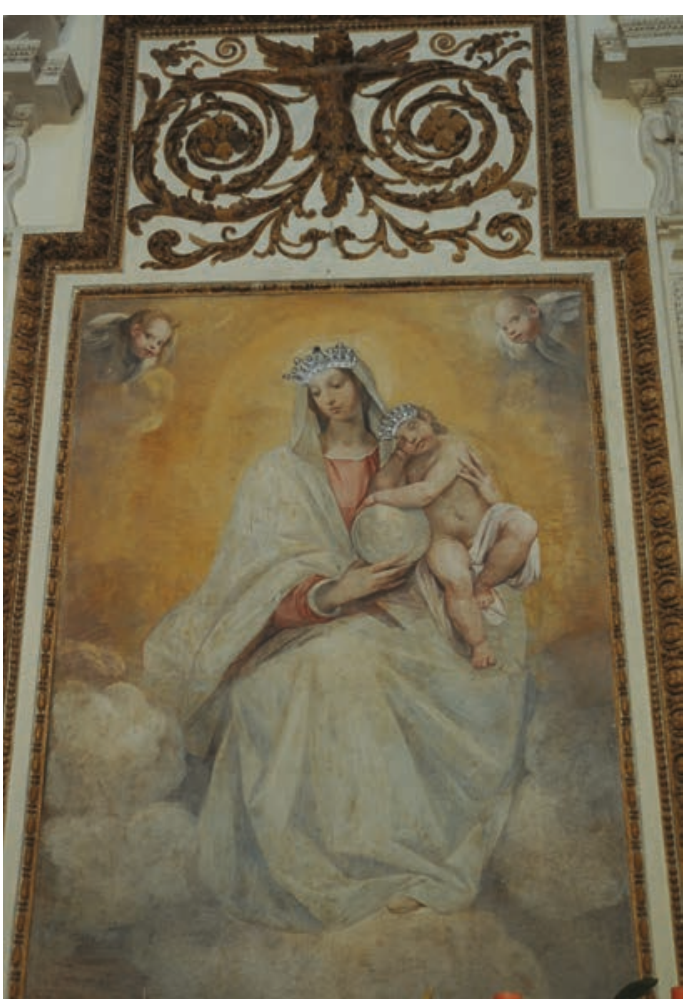

Fig. 2. Giuseppe Cesari. Virgen con el Niño dormido. Colegiata de San Lorenzo en Zagarolo, Roma.

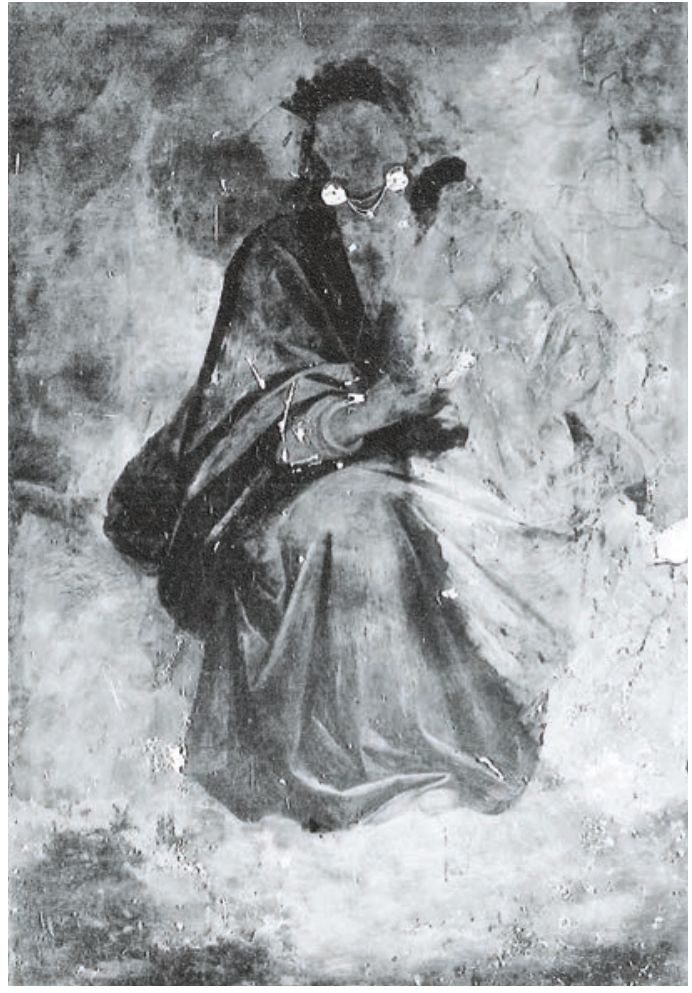

Fig. 3. Giuseppe Cesari. Virgen con el Niño dormido. Antes de su restauración. Colegiata de San Lorenzo en Zagarolo, Roma.

de $1812^{5}$, si bien es cierto que en marzo de 1811 se publicó en la Gazeta de Madrid una recensión, escrita por cierto "J. A.", sobre la primera parte de la Histoire de l'Art de Seroux d'Agincourt, que los estudiaba ${ }^{6}$. Así, en julio de 1817 Carlos Miguel compraba en Florencia por 448 francos una Madonna de Fra Angelico que hoy se encuentra en el Museo del Prado, y por 336 francos una pequeña tablita atribuida a Masaccio hoy en la misma institución ${ }^{7}$. Cifras entre las más bajas empleadas en la compra de pintura, y que corresponden al interés todavía relativo hacia los primitivos, cuya inclusión en la colección se supone más por la intención de reflejar, al modo del Musée Napoleon, una visión enciclopédica de la Historia del arte, que por otras consideraciones.

El cartón del Palacio de Liria se relaciona con el fresco de la Virgen con el Niño dormido de la Colegiata de San Lorenzo en Zagarolo que Röttgen atribuyó a Cavalier d'Arpino (fig. 2), repintada torpemente en su parte derecha y que había perdido el fondo, el rostro y buena parte de los ropajes de la Virgen, tal y como evidencia la fotografía amablemente proporcionada por el estudioso (fig. 3) ${ }^{8}$. El mural ha sido restaurado recientemente y, suponemos, pues no ha sido

${ }^{5}$ La marquesa de Ariza, su madre, inicia su diario de viaje e la capital francesa el 15 de abril de ese año. Marie Thérèse de Silva y Palafox. Notes de mes voyages en Italie, 1812-1814, ADA 345.12.

6 Gazeta de Madrid, 15 de marzo de 1811.

7 El hallazgo de un libro de cuentas que recoge de manera minuciosa los gastos de Carlos Miguel durante un periodo de 29 meses entre 1816 y 1818 corrobora estas cifras. La transcripción e interpretación de estas cuentas forma parte de un trabajo, ahora en prensa, que hemos dedicado a la figura del coleccionista. Compte Général des Recettes et des Dépenses faites pour le Soussigné d'ordre et pour compte de Son Excellence Monsieur le Duc de Berwick et d'Albe. Bibliothèque Nationale de France, Département des manuscrits, NAF 21193. Compte f. 6 v., 8 de abril de 1816. Las cantidades son referidas en francesconi y francos.

${ }^{8}$ Röttgen, 2003: 390 


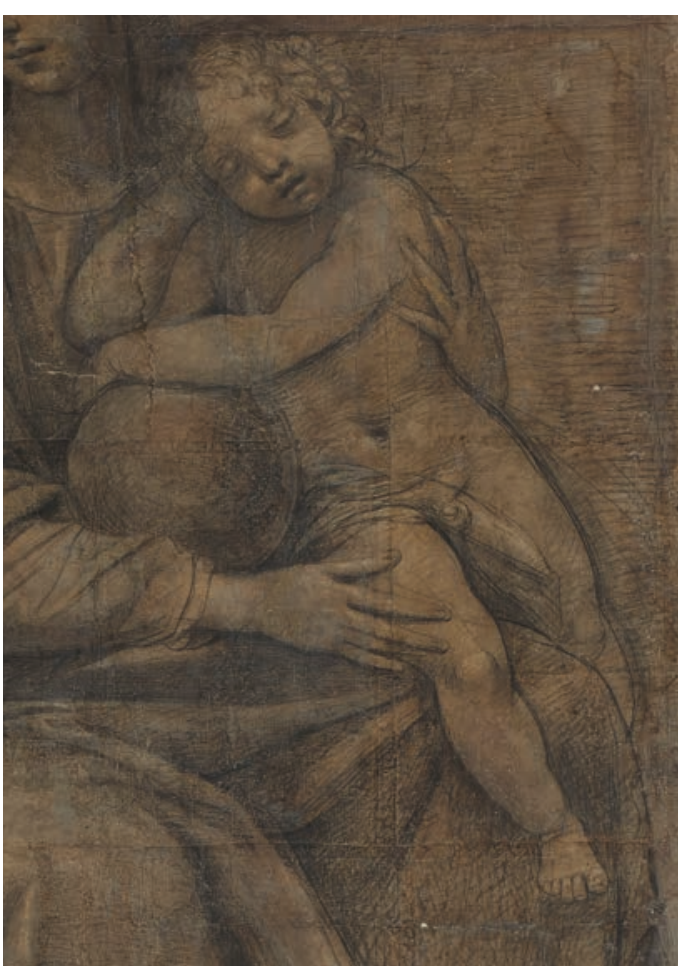

Fig. 4. Giuseppe Cesari. Virgen con el Niño dormido, detalle. Colección Casa de Alba. Palacio de Liria. Madrid. posible acceder al informe de restauración, que las partes en las que se había desprendido la capa pictórica habrán sido reconstruidas según las incisiones todavía existentes sobre el intonaco (fig. 2) ${ }^{9}$. Así, el rostro de María en el dibujo se corresponde notablemente, incluidas la sombras bajo el mentón y los párpados, con el que ha sido recreado tras la restauración. Por otro lado, el torso y la pierna derecha de Cristo Niño y el extremo derecho del manto de María repiten con exactitud las líneas del cartón, que recoge la figura de la Virgen de la cabeza a los pies y mide 194 x $139 \mathrm{~cm}$, mientras que el fresco mide $300 \times 170 \mathrm{~cm}$, si bien incluye espacio a ambos lados del grupo $\mathrm{y}$, más aún, sobre y bajo María ${ }^{10}$. Aunque no ha sido posible medir la figura de María en Zagarolo, su tamaño parece ser el mismo.

El cartón no presenta ninguna señal de transferencia y su composición muestra variantes muy vistosas respecto al fresco: como la posición del brazo y la mano derecha de la Virgen, que en el fresco sostiene el orbe, o una de las piernas de Cristo niño, que aparece recogida. Así pues, se trataría de un "ben finito cartone", mientras que en Zagarolo se habría empleado otro cartón, derivado probablemente del que aquí se presenta, para realizar el cal-

co $^{11}$. El término "ben finito cartone" no se refería en el siglo XVI tanto al acabado de la obra como al efecto de "rilievo" logrado por medio del claroscuro; entre otros usos, este cartón podía servir como guía cuando la ejecución final era delegada ${ }^{12}$. Este parece el caso del fresco de Zagarolo, que sigue en buena medida el dibujo de la Casa de Alba, en el que se puede apreciar, a la derecha de la cabeza de Jesús, el rostro socarrón de un niño que no remite directamente a ninguna pintura o dibujo conocido de Cesari, quien, sin cerrar los contornos de las figuras (como se puede comprobar en los brazos y pies del niño o en las manos de su madre) emplea una línea vibrante, pero decidida, que le permite encajar la composición de manera definitiva, sin excluir alguna variante (fig. 4).

La datación del cartón y del fresco resulta complicada. Röttgen nota que una visita apostólica realizada en 1754 atribuye la pintura a Cavalier d'Arpino y que cierta Francisca Bonafede había dado orden en su testamento redactado en 1608, de celebrar una misa cantada anual en la capilla donde se encuentra, lo que situaría la decoración hacia 1610, pero por otro lado nota que el rostro de María remite a obras tardías de Cesari como la Madonna con el niño, San

9 En el momento de escribir esta nota no ha sido posible acceder al informe de restauración de la obra. Las fotografía de la pintura y la información sobre las dimensiones del fresco han sido proporcionadas amablemente por el arquitecto Agapito Fornari, director de la Oficina de bienes culturales de la diócesis de Palestrina. Quiero dar las gracias a las funcionarias de la Soprintendenza de Roma Dora Catalano, Isabella del Fratte y Maria Castellino por su ayuda para obtener una fotografía de la pintura.

${ }^{10}$ Las dimensiones del fresco figuran en BeWeb (Inventario dei beni storici artistici delle diocesi italiane). https://www.beweb.chiesacattolica.it/benistorici/bene/4745288/Cesari+G.+sec.+XVI\%2C+Dipinto+murale+con+la+ Madonna + del + Carmelo\#locale $=$ it\&action $=$ CERCA\&da $=11 \&$ frase $=$ cavalier+arpino\&ordine $=$ rilevanza Consulta realizada el 5-10-2017.

11 Sobre los cartones véase el ejemplar estudio de Bambach, 1999: 249-276 y 277-295.

12 Bambach, 1999: 264-272, con la bibliografía precedente. 


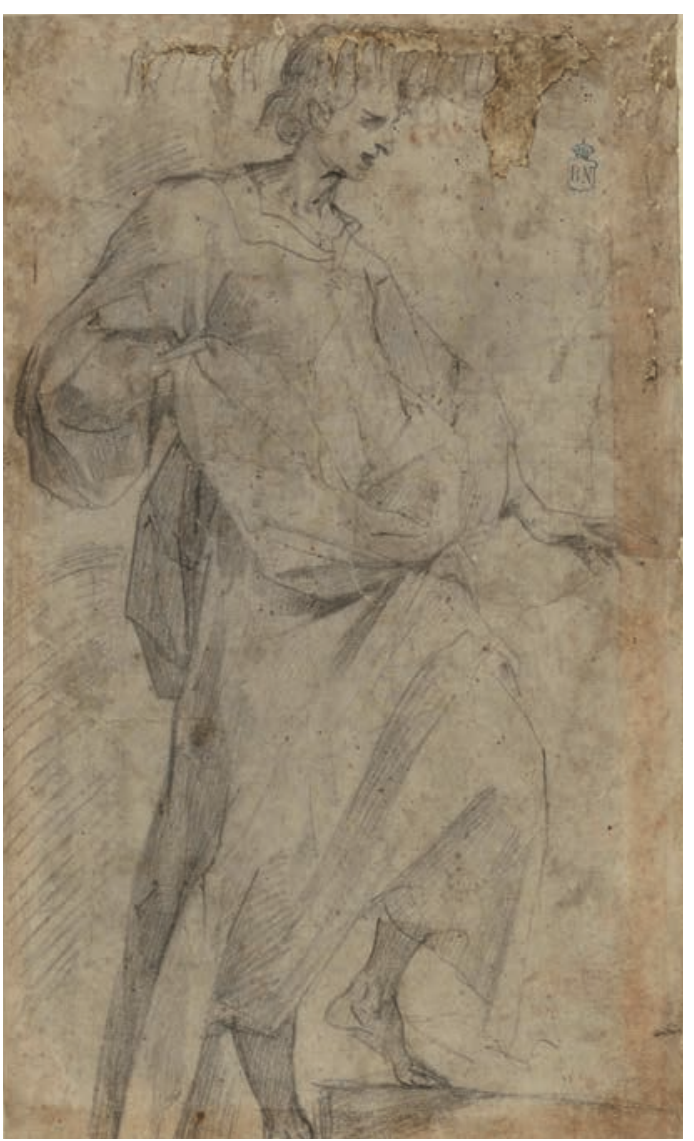

Fig. 5. Giuseppe Cesari. ¿Figura de apóstol?. BNE, DIB/16/39/14.

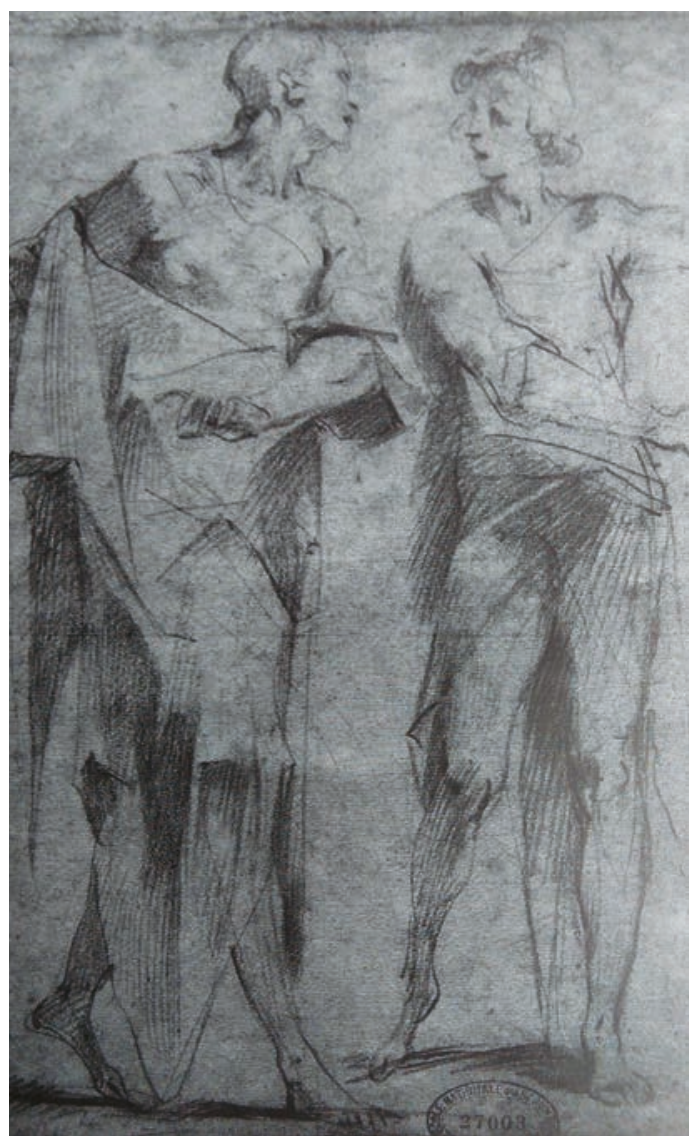

Fig. 6. Giuseppe Cesari. ¿Pareja de apóstoles?. Ècole nationale supérieure des Beaux-Arts. París.

Francisco y San Honorio de S. Lorenzo in Fonte de Roma, hoy en la catedral de Fiuggi, por lo que fecha la pintura hacia 1630-35 ${ }^{13}$. Calenne se remite a una visita apostólica efectuada en 1620 que señala la capilla bajo la advocación de la Virgen del Carmelo e informa de que cierto "Astorrem Palatium" (al que podría corresponder el escudo en piedra en su interior, cuyo campo recuerda a un palacio fortificado) se había comprometido a mantenerla; sin embargo, una visita fechada en 1659 menciona una "Imago B.me Mariae Filium gestantis" pintada sobre el muro por "Equitis Josephi Arpinatis cum ornamento ex stucco inaurato"14. La similitud iconográfica con la Madonna para el techo de San Crisógono de Roma, documentada en 1622, y con la perdida Madonna de la catedral de Sora (que Röttgen vio en fotografía y relacionó con el fresco de Zagarolo), junto con el hecho de que Ottavio Grisolati, al que Calenne atribuye convincentemente la decoración en estuco de la capilla, había trabajado a menudo para Cesari entre 1622 y 1626, han llevado a este último a fechar el fresco en los primeros años de esa década ${ }^{15}$, algo muy razonable.

13 Röttgen, 2002: 392 y 467

14 Calenne 2010: 190-194. Así pues, parece que la capilla estaba inicialmente bajo la advocación de María del Monte Carmelo y que en algún momento entre 1620 y 1659 su nuevo propietario cambió su dedicación y encargó su decoración con un fresco y estucos. Aunque sería necesario explicar por qué la visita de 1754 insiste en la vieja iconografía del Carmelo (no era raro, en cualquier caso, repetir sumariamente lo referido en anteriores visitas).

15 Calenne, 2010: 190. Sobre el cuadro de San Crisógono y el de Sora, Röttgen, 2002: 431y 507. Sobre el trabajo de Grisolati para Arpino, Calenne, 2010. 


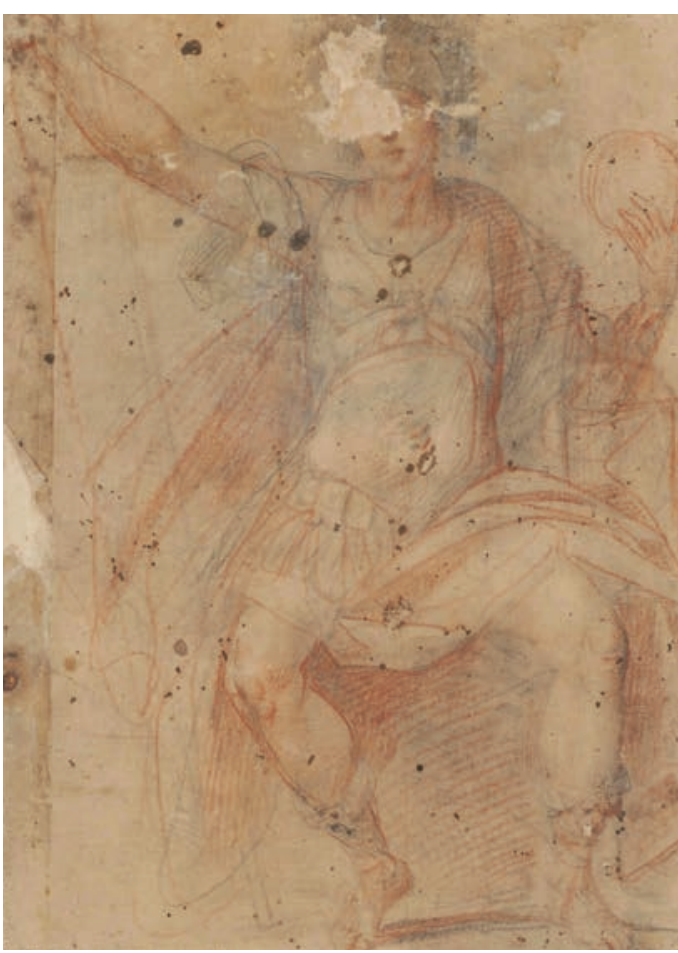

Fig. 7. Círculo de Giuseppe Cesari. ¿Un emperador?. BNE, DIB/15/15/22.

Por lo que respecta al momento de ejecución del cartón Alba, la comparación con las obras de Arpino en el mismo formato no ayuda demasiado. Los cartones realizados duran- te la primera década del siglo XVII para los ángeles en mosaico de la cúpula de San Pedro - de unos $350 \times 150 \mathrm{~cm}$ - eran muy diferentes pues las figuras debían ser vistas a gran distancia, lo que exigía simplificar sus formas ${ }^{16}$. La estrecha relación del dibujo con el fresco de Zagarolo invita a fecharlo también en la década de 1620; por lo demás, la tipología del rostro de María - reducido en los cuadros de cierto tamaño a un esquema geométrico muy reconocible desde aproximadamente 1615 hasta la muerte de Cesari- ${ }^{17}$ es muy similar al rostro de María en la pintura del techo de San Crisógono, documentada en $1622^{18}$.

La Biblioteca Nacional conserva un dibujo a lápiz negro que Barcia atribuyó a El Greco y que Angulo y Pérez Sánchez consideraron obra de un anónimo español del siglo XVII (fig. 5) ${ }^{19}$. Según indica una anotación en la fotografía del mismo, sobre el antiguo montaje del folio - no visible en la actualidad- existía una nota en la que Pérez Sánchez (al que se identificaba por su caligrafía), sugería que el dibujo "podría ser" de Cavalier d'Arpino. Las recientes monografías de Bolzoni y Röttgen sobre la obra gráfica del pintor permiten corroborar esta sugerencia ${ }^{20}$.

Efectivamente, el alargado canon de la figura, su rostro enjuto y aquilino, el corte anguloso y afilado de su vestimenta y el característico empleo de los trazos en líneas verticales que se encuentra en otros dibujos juveniles del pintor, bastan para asignarle el folio. La comparación más precisa se puede establecer con una sanguina custodiada en la Escuela de Bellas Artes de París atribuida por Röttgen (fig. 6) ) $^{21}$ en la que Bolzoni nota las características estrías verticales de su sombreado (casi una firma del artista, en sus palabras), y que ha relacionado - siguiendo a Blunt y Pouncey - con el dibujo del Museo Fitzwilliam de Cambridge que presenta la parte izquierda del destruido fresco San Lorenzo entre los pobres y enfermos, pintado por Cesari para San Lorenzo in Damaso en Roma entre 1588 y $1589^{22}$. El característico estilo del dibujo madrileño se encuentra también en el Hombre sentado atribuido a Cesari por Bolzoni en el Herzog Anton Ulrich-Museum, o en otro que considera, con razón, una variante para las dos figuras que aparecen sentadas en la parte derecha del folio

\footnotetext{
16 Empresa ejecutada entre 1603 y 1612. Röttgen 2002: 353-364 cat. 115.

17 Röttgen, 2002: 154.

18 Röttgen, 2002: 431 y 467. Calenne, 2010: 190-191.

19 Inv DIB/16/39/14, la ficha de inventario señala que se trata de papel verjurado amarillento de 170 x $288 \mathrm{~mm}$ y que el dibujo fue pegado a un segundo soporte y este a un tercero; el folio fue adquirido en 1899 y procede de la colección Madrazo. Angulo, Pérez Sánchez, 1977, I: 44.

${ }^{20}$ Röttgen, 2012-2013.

21 Bolzoni, 2013.

22 Sobre estos dibujos, con la bibliografía previa Röttgen 1973: 148. Röttgen, 2012-2013, I: 244, 246. Bolzoni, con toda la bibliografía precedente, aprecia en ellos la influencia de Vanni y Salimbeni, a los que Cesari frecuentó durante el "cantiere" de Sixto V. Bolzoni, 2013: 171, 173.
} 


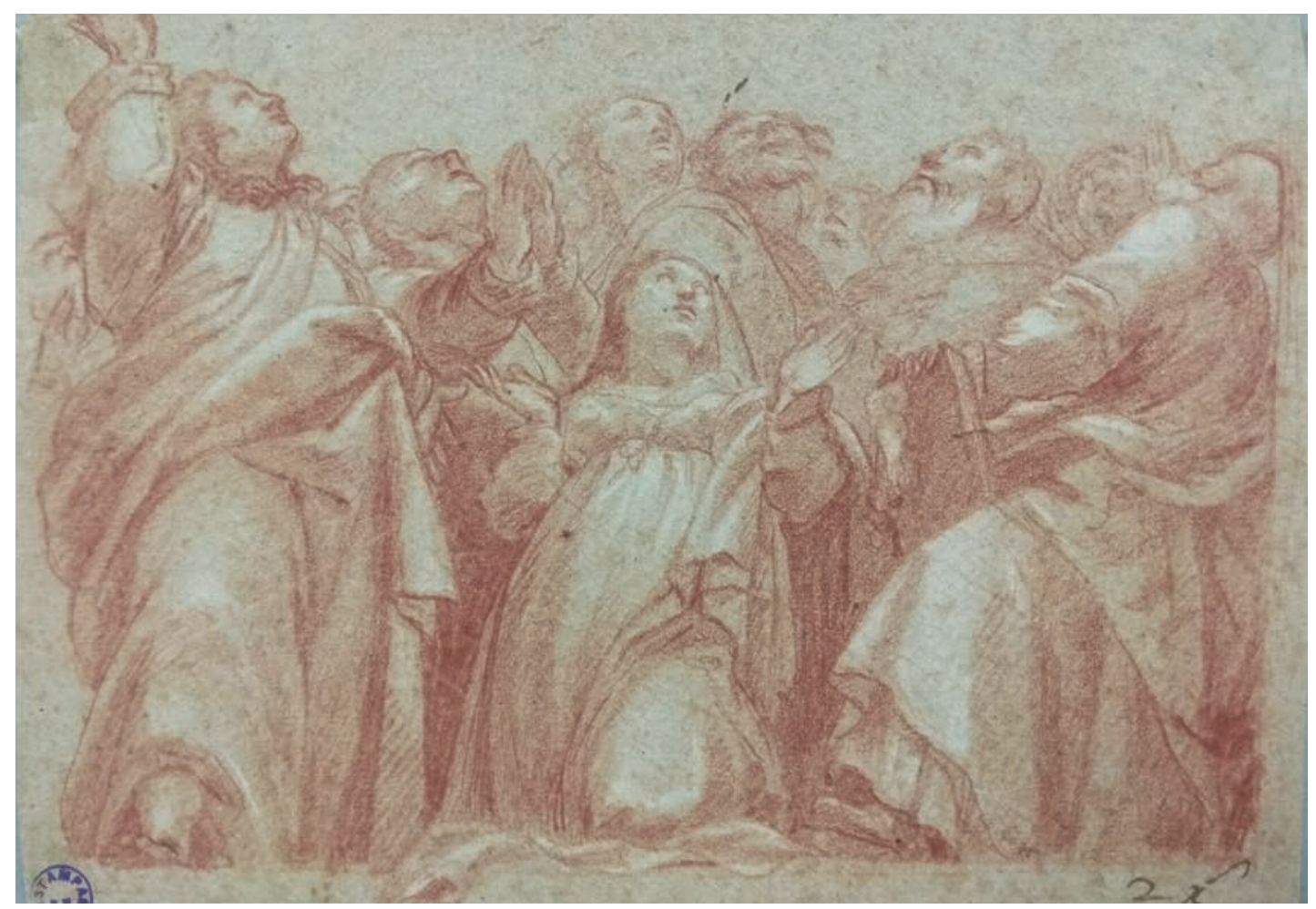

Fig. 8. Anónimo (de Giuseppe Cesari). BNE, DIB 18/1/1375.

de Cambridge ${ }^{23}$. También se puede mencionar como termino de comparación el dibujo para la Resurrección de Lázaro en la Albertina, fechado hacia 1592-324, que muestra en la figura de Cristo rasgos comunes con el personaje de la Biblioteca Nacional, cuyo perfil recuerda, simplificado, al que aparece en dibujo conservado en los Uffizi realizado por las mismas fechas para una figura de la capilla Contarelli ${ }^{25}$. No obstante, la frágil anatomía y el pequeño tamaño de la cabeza de la figura madrileña invita a precisar su ejecución entre 1588 y 1590 , dada su estrecha relación con los folios para la decoración de San Lorenzo in Dámaso ${ }^{26}$.

La Biblioteca Nacional custodia un dibujo que parece representar a un emperador y que Jaffé atribuyó en 1960 a Cavalier d'Arpino (fig. 7), opinión corroborada por Rötgen, que lo considera uno de los primeros folios del pintor, realizado hacia 1582-8527. Sin embargo, Bolzoni lo ha incluido en su catálogo en el apartado dedicado a los dibujos del taller o la "cerchia" del artista ${ }^{28}$. Algo que suscribimos: a nuestro parecer, la manifiesta incorrección en la representación del escorzo del brazo recogido de la figura es difícilmente justificable, incluso teniendo en cuenta la posible juventud del pintor, por lo que debe tratarse de un producto de su taller.

23 Braunschweig, Herzog Anton-Ulrich Museum, inv. Z 1629. El segundo fue atribuido por Röttgen en ocasión de su venta en 1981. Bastille, 1981: cat. 30. Bolzoni, 2013: 173, 178.

24 Röttgen, 2012-2013, I: 353. Bolzoni, 2013: 204-4, 216-217.

25 Sobre este, Röttgen 2012-2013, I: 356. Bolzoni, 2013: 214.

${ }^{26}$ El folio fue adquirido en 1899 a Álvaro Gil y Albacete, en 1899 y procedía de la colección de José de Madrazo, que lo pasó en un lote a su hija, que a su vez lo cedió a su hijo Isidoro Gil y Madrazo, hermano consanguíneo del citado. Barcia 1906: 7.

27 La opinión de Jaffé fue anotada sobre el paspartú, donde B.W. Meijer insiste en la atribución al pintor.

Biblioteca Nacional DIB/15/15/22. Lápiz negro, lápiz rojo, 236 x 169 mm. Röttgen, 2012-2013, III: 52, cat. 14.

28 Bolzoni, 2013: 422. 
La institución conserva un folio que copia la parte inferior de la Asunción de María pintada al fresco por Cavalier d'Arpino en la capilla Olgiati (fig. 8). En el verso de su montaje se ha escrito a lápiz "Corn Saftleven", razón por la que el catálogo de la Biblioteca Nacional lo asigna al pintor holandés Cornelis Saftleven (Gorinchen 1607-Rotterdam 1681) ${ }^{29}$. El dibujo es una transcripción literal, a pesar de ser sumaria, de todos los detalles pintados por Cesari, con cuyo estilo gráfico no guarda relación alguna ${ }^{30}$. Que se sepa, Saftleven nunca visitó Italia, pero dibujos como la mujer a la carrera, monografiado y fechado $1632^{31}$, demuestran una técnica similar, por lo que la atribución al pintor es adecuada.

\section{BIBLIOGRAFÍA}

Almanach Royal presenté à Sa Majesté par Testu (1829): París, Guyot et Scribe.

Amadio, Sonia (2007): "Carlo Baldeschi, Jean-Baptiste Wicar, Tommaso Minardi e alcuni disegni di fra' Bartolomeo", Les cahiers d'Histoire de l'art, 5, Roma, pp. 71-76.

Angulo, Diego / Pérez Sánchez, Alfonso Emilio (1977): A Corpus of Spanish Drawings, I, Londres: Harvey Miller.

Bambach, Carmen (1999): Drawing and Painting in the Italian Renaissance Workshop: Theory and Practice, 1300-1600, Cambridge: Cambridge University Press.

Barcia, Ángel María de (1906): Catálogo de dibujos originales de la Biblioteca Nacional, Madrid: De la Cuesta.

Bolzoni, Marco Simone (2013): Il Cavalier Giuseppe Cesari d'Arpino: maestro del disegno. Catalogo ragionato dell'opera grafica, Roma, Bozzi.

Calenne, Luca (2010): Prime ricerche su Orazio Zecca da Montefortino (oggi Artena): dalla bottega del Cavalier d'Arpino a quella di Francesco Nappi, Roma: Gangemi.

Förster, Sabrina (1992): Meisterzeichnungen 1590-1900, Gallerie Sabrina Förster, 22, Dusseldorff, 1992.

Gazeta de Madrid, 15 de marzo de 1811.

Houliston, Laura (2012): The Suffolk Collection, Swindon: English Heritage.

De Francisco Olmos, José María (2001): “Conflictos bélicos y circulación de moneda extranjera en España 1808-1836. La documentación de la inestabilidad”, Revista General de Información y Documentación, 11, I, pp. 109-131.

Pita Andrade, José Manuel (1959): El Palacio de Liria, Madrid: Instituto de Estudios Madrileños.

Röttgen, Herwarth (1973): Il Cavalier d'Arpino, Roma: De Luca.

Röttgen, Herwarth (2002): Giuseppe Cesari d'Arpino, un grande pittore nello splendore della fama e nell'inconstanza della fortuna, Roma: Bozzi.

Röttgen, Herwarth (2012): Giuseppe Cesari d'Arpino. Die Zeichnungen. I-III, 13, Stuttgart: Opus Magnum.

Schulz, Wolfang (1978): Cornelis Saftleven 1607-1681, Leben und Werke, mit einem kritischen Katalog der Gemälde und Zeichnungen, Berlin, New York: Walter de Gruyter.

Fecha de recepción: 09-IX-2018

Fecha de aceptación: 19-XII-2018

${ }^{29}$ BNE DIB 18/1/1375. Transcribimos lo reportado en el inventario online de la BNE: "Sanguina sobre papel verjurado crema. Inscripción manuscrita a tinta en el ángulo inferior derecho del recto "2 rs". Inscripción manuscrita a tinta en el ángulo inferior derecho del recto del soporte secundario "329", 248 x 184 mm. Sobre Saftleven Schulz 1978.

30 Sobre los dibujos para la capilla Olgiati, Bolzoni, 2013: 240-241; Röttgen, 2012-2013, II: 50-54. Por último, cabe mencionar dos pinturas que se pueden adscribir también al taller de Arpino, sin que sea posible, por el momento, especificar su autor. Una de ellas se encuentra en colección privada en Madrid. Se trata de una Resurrección de Lázaro que repite la pintura del mismo tema en la Galleria Corsini y que en palabras de Röttgen (comunicación oral tras la visión de una fotografía) es la mejor réplica de la misma, con la que comparte su rica pincelada pero carece de su calidad cromática; algunos rostros, como el de Magdalena, arrodillada, remiten a la labor del taller de Cesari después de 1620, y pudo haber sido pintada por Bernardino o Muzio Cesari. La otra pintura, de pequeño formato, se encuentra en la Suffolk Collection, donde está atribuida a un anónimo italiano del siglo XVI, pero como es manifiesto, retoma la Visitación pintada por Cesari hacia 1605-6 para la capilla del obispo de Narni Giovan Battista Toschi en la catedral de Reggio Emilia y el gran cuadro del mismo tema, pero con la composición invertida y algunas variantes, del altar mayor de la catedral de Narni, también fechado por entonces. La pintura inglesa se podría colocar antes, a finales en la década de 1590, en atención a sus tipos, menos severos. La presencia de la figura de la dama con el cabello ricamente peinado, un tipo completamente ajeno a Cesari, sugiere un posible retrato, ligado tal vez, a la comisión de la pintura. Sobre las obras de Reggio Emilia y Narni, Röttgen, 2002: 377-378. Sobre el lienzo inglés Houliston, 2012: 292-293. Oleo sobre tabla 57.2 x 46,5 cm inv. 88019294.

31 Förster, 1992, p. 6. 